See discussions, stats, and author profiles for this publication at: https://www.researchgate.net/publication/326793557

\title{
Mental Disorder as Both Natural and Normative: Developing the Normative Dimension of the ze Conceptual Framework for Psychopathology
}

Preprint in Journal of Theoretical and Philosophical Psychology · January 2019 DOI: $10.1037 /$ teo0000118

\section{CITATIONS}

4

2 authors:

Kristopher Nielsen

Victoria University of Wellington

9 PUBLICATIONS 26 CITATIONS

SEE PROFILE
1,065

(8) Tony Ward

Victoria University of Wellington

476 PUBLICATIONS 19,910 CITATIONS

SEE PROFILE

Some of the authors of this publication are also working on these related projects:

Determining Sexual Offender Motivational Systems View project

The role of detained female adolescents' quality of life in explaining offending outcomes in emerging adulthood (Postdoc) View project 
(C) 2019, American Psychological Association. This paper is not the copy of record and may not exactly replicate the final, authoritative version of the article. Please do not copy or cite without authors' permission. The final article will be available, upon publication, via its DOI:

$10.1037 /$ teo0000118

Mental Disorder as both Natural and Normative: Developing the Normative Dimension of the 3 e Conceptual Framework for Psychopathology

Kristopher Nielsen

Victoria University of Wellington

Tony Ward

Victoria University of Wellington

Ghent University

Corresponding Author:

Kristopher Nielsen

School of Psychology

Victoria University of Wellington, PO Box 600.

Wellington, New Zealand.

E-mail: Kristopher.Nielsen@vuw.ac.nz

Tony Ward: Tony.Ward@vuw.ac.nz 


\begin{abstract}
Current arguments concerning the role of normativity within the concept of mental disorder are explored, and some requirements of a successful normative construal sketched out. We then shift to a discussion of "natural" normativity in order to lay the groundwork for our own understanding of what counts as mental disorder. The view we present is grounded in an enactive, embodied, and embedded view of the mind ( 3 e cognition). The position argued for is one where the labeling of a particular set of behaviors as disordered or dysfunctional is justified by the significant violation of norms, but where the norms in question are not socially imposed but are the functional norms of the individual being diagnosed. The strengths and weaknesses of our position are discussed, and an addendum proposed in response to a foreseeable counter-argument. This construal provides a conceptual framework for thinking critically about normative issues in diagnosis, appreciates how central normativity is to the concept of mental disorder, and finally (in being tied to the functionality of the individual), places the institutions of psychiatry and clinical psychology on good ethical ground and allows for consideration of cultural and individual variation during the diagnostic process.
\end{abstract}

Keywords: 3e; psychopathology; norms; values; embodiment 


\section{Introduction}

In the 1960's and 70’s Thomas Szasz famously made the claim that mental disorder was a myth. Specifically, he claimed that the diagnoses of psychiatry were at best 'problems in living', at worst merely labels given to those that break the normative mold of society (Szasz, 1960, 1963, 1974). The assertion that mental illness is socially constructed strongly resonated with the anti-psychiatry movement, whose influence is still evident today (Lieberman \& Ogas, 2015). Researchers and clinicians currently working in mental health arguably need to respond to such claims and understand the role of normativity in diagnosis and practice. The central questions are: what role if any should norms and values play in deciding what counts as mental disorder? what kinds of norms should play this role? and whose norms are of interest?

At the outset we should specify that we are primarily concerned with what is normatively required for something to be considered a mental disorder, and largely set aside ontic and epistemological issues. Broadly, the current paper aims to first explore arguments concerning the role of normativity within the concept of mental disorder and to sketch out some requirements of a successful normative concept of mental disorder. Following the listing of these requirements we shift to a discussion of "natural" normativity and the deep continuity thesis of life and meaning. This is necessary in order to lay the ground work for our own understanding of what counts as mental disorder, which we will subsequently explicate. The view we present is grounded in an enactive, embodied, and embedded view of the mind (3e cognition) ${ }^{1}$. Within this article we do not seek to defend $3 \mathrm{e}$ Cognition, but rather seek to explore its implications within this area. The strengths and weaknesses of our position are

\footnotetext{
${ }^{1}$ See in the $3 e$ cognition section as to why we do not subscribe to extension/4e.
} 
discussed, and an addendum proposed in response to a foreseeable counterargument. In the concluding section, we summarize and briefly explore the ramifications of our analysis for the development of a 3 e conception of mental disorder, a larger project of which this article is part (Nielsen \& Ward, 2018a, 2018b).

To foreshadow our general conclusions, the position argued for is one where the labeling of a particular set of behaviors as disordered or dysfunctional is justified by the significant violation of norms, where the norms in question are not socially imposed but rather are the functional norms of the individual being diagnosed. Significance, in this context, is conceived of in reference to the degree to which the self-maintenance and adaption of the organism is threatened. These two concepts are borrowed directly from $3 \mathrm{e}$ Cognition. We will attempt to show how this construal provides a conceptual framework for thinking critically about normative issues in diagnosis, appreciates how central normativity is to the concept of mental disorder, and finally (in being tied to the functionality of the individual), places the institutions of psychiatry and clinical psychology on good ethical ground and allows for consideration of cultural and individual variation during the diagnostic process.

\section{Recent Views on the Role of Normativity}

There has been previous debate in the research literature about the role that values should play in diagnosis. Most generally this has been a two-sided argument in the form of 'values in' (evaluativist) versus 'values out' (descriptivist) positions. The former position arguing that the evaluative nature of a diagnosis is inescapable, while the latter proposes that diagnostic claims are purely factual in nature (Fulford, 2002). The question of whether norms and values have a role to play at all is somewhat trivial; at its simplest, a diagnosis is a claim that something is wrong with a person. On our view it is therefore necessarily normative, and we therefore assume 
an evaluativist position within this paper (others do disagree; see Hucklenbroich, 2014). The more interesting question seems to be around what kinds of norms and values demarcate disorders from benign conditions, and how should they be employed to do so. Particularly contentious is the question of whether social and cultural norms should play a role (strong evaluativism) or not (weak evaluativism).

In what follows, we sample some current and representative work in this area. There is a large and disparate literature that pertains to this issue and it is therefore not feasible to perform a complete review (for relevant readings see: Abouelleil \& Bingham, 2014; Bolton, 2008; Browne, 2017; Fulford, 2001, 2002; Hucklenbroich, 2014; Kendler, 2016; O'Connor, 2017; Porter, 2010; Sadler, 2005; Stein et al., 2010). Rather, in order to streamline our discussion in this section, we concentrate on an article by Stier (2013) and a selection of responses. We have chosen this formulation because it manages to capture the core issues at play in a succinct manner. Our aim is to draw out what is required of a framework attempting to conceptualize the role of normativity in demarcating mental disorder.

\section{Sample of Work in this Area}

Stier (2013) makes the claim that with the progression of neuroscience the medical model is gaining increasing traction within psychiatry. With the rise of a biologically based psychiatry Stier argues that we are disregarding the obviously normative nature of assessing human behavior and making diagnostic claims. On his view, on the basis of growing knowledge of the brain we mistakenly conclude that disorder itself is always reducible to a brain abnormality. Even if we assume that all behavior and experience stems from the brain (a counter-embodiment position he assumes within the context of his argument), the label of 'disorder' relies on assessment of the experience and behavior of the individual as pathological. Mental 
disorder therefore, cannot be identified at a purely physiological level. According to Stier, there are many normative frames of reference against which psychiatry makes a diagnostic judgement: the personal values of the diagnostician, cultural expectations, generalizations about human nature, and the concepts of harm and disturbance. The examples he uses suggest a strong form of evaluativism (i.e. one inclusive of social and cultural norms). Stier goes on to explore some further normative concepts that play vital roles in psychiatry, but for the purposes of this discussion what we have covered here will suffice. Stier concludes that the prevalence of such normative factors within psychiatry as a practice supports his earlier argument that whether or not something is a mental disorder can only be determined on the mental (including behavioral) level.

Responding to Stier's (2014) claims, Muders (2014) raises two key criticisms. First, that Stier seems to be talking about the practice of psychiatry as it is done, rather than arguing for how it should be done. In doing so, he misses the possibility that while we currently rely on these normative frames of reference, this may actually be an error and thereby not suggestive of what the concept of mental disorder should be. Second, Muders suggests that Stier fails to unpack what it means for something to be normative. While the position we will eventually argue for is in line with Stier's claim regarding the irreducibility of mental disorder to a brain state, Muders criticisms are valid. A framework circumscribing the role of normativity in the concept of mental disorder needs to be clear about what kind of norms are at issue and where they come from. It should also make a distinction between the concept as evident in the current process of diagnosis and the 'ideal' concept- how mental disorder should be thought of. Regarding this last point, we wish to make it clear 
that throughout this paper we are attempting to aim for the later; to develop a concept of what diagnosis should ideally represent within the normative domain.

Jefferson (2014) also responds to Stier (2014). In the second half of her paper, she turns to the role that Stier describes normativity as having in the act of diagnosis, highlighting that his position is more than mere weak evaluativism. Rather, Stier's assertion is that diagnostic claims in psychiatry are directly and pervasively influenced by moral, social, and cultural norms - a strong form of evaluativism thereby introducing a large degree of relativity. Jefferson argues that this is problematic because it does not seem acceptable that what counts as disorder in one culture changes if somebody was uprooted to another culture with differing standards. While she accepts that some degree of vagueness is inescapable, Jefferson argues that we should strive for objectivity in diagnosis. She calls for “...a standard according to which we judge whether calling a certain condition pathological is valid or not." (Jefferson, 2014, p. 2).

While not directly responding to Stier (2014), Banner (2013) makes points relevant to the task at hand. She argues that mental disorders cannot be completely reduced to brain disorders on the basis that if a brain abnormality does not lead to a problem at the mental/behavioral level then it is not a mental pathology. Rather, the label 'mental disorder' indicates a problem at the level of the person functioning in their environment ${ }^{2}$. While some mental disorders have been found to correlate with abnormalities at a brain level, Banner correctly points out that it is the dysfunction at the level of the person that makes it pathological, not its (partial) instantiation in the brain. While the general thrust of Banner's position is parallel to Stier's claims

\footnotetext{
${ }^{2}$ While not part of the sampled debate, Frisch (2014) makes very similar points based on an exploration of the ideas of Kurt Goldstein, one of the founders of clinical-neuropsychology. He demonstrates that Goldstein's ideas were remarkably similar to what we express in this article.
} 
around irreducibility, there are two elements of Banner's construal that are particularly interesting. Firstly, she highlights the role of the social and environmental context in shaping what counts as disordered. Secondly, she defines mental disorder as specifically concerned with deviation from the individual's functional norms; those norms that support the functioning of persons within their context ${ }^{3}$.

\section{What Can Be Learnt}

Out of this discussion, and most clearly implied by Muders (2014), two key requirements emerge: A normative concept of mental disorder must be clear about, 1) what kind of norms are at issue, and 2) where they come from.

Regarding the first requirement, it should be apparent that the most contentious question is whether or not socio-cultural norms have a role to play in demarcating mental disorders from benign conditions. Classically speaking, if they do then this would constitute a strong evaluativist position. If they do not, and the norms in question are simply those of the individual, then this would constitute a weak evaluativist position. However, we argue that this way of discriminating positions represents unduly dichotomous thinking. To explain this, we will first briefly summarize some of the positions of the papers explored above.

Stier (2013) suggests that psychiatry is currently acting on an implicit strong evaluativism, but does not really comment as to whether or not this is justified. Jefferson (2014) in contrast, correctly points out that incorporating socio-cultural norms into the concept of diagnosis leaves us in an uncomfortably relativistic

\footnotetext{
${ }^{3}$ Banner further divides these functional norms into separate domains within which they may fall, namely: epistemic, rational, emotional, moral, social, and those concerning self-knowledge. We don't delve into these distinctions here but they are potentially quite useful.
} 
position whereby disordered status may completely change with shifts in cultural contexts. This would position Jefferson as weakly evaluative. Banner (2013) however does something quite different. She emphasizes the functionality of the individual within their social and environmental context and defines mental disorder upon the breaking of the norms that support this functionality. At a first glance, this may seem to be a strongly evaluative position because, given that the socio-cultural environment plays a large role in deciding whether an action is functional or not, it leads to a situation where what counts as disordered changes with cultural and situational context ${ }^{4}$. On the basis of this, it appears that Banner's position is strongly relativistic.

However, on further inspection Banner's (2013) position is a lot more nuanced than this, and indeed, more nuanced than she explicitly recognizes in her original paper. In being based on functional norms, her move allows only those socio-cultural norms that are crucial for the continued adaptive functioning of the individual within their context, while excluding those norms that merely serve the group or are merely statistical. While not explicitly stated, some socio-cultural norms are let in, and some are not, based on whether or not they contribute to functioning of the individual. This then begins to move beyond the classic dichotomy between weak and strong evaluativist positions. Further, this leaves the act of diagnosis as justifiable purely by reference to supporting the individual, thereby countering Szaszian type claims without ignoring the role of culture and context. When

\footnotetext{
${ }^{4}$ And indeed it does seem to, for example, Fulford and Jackson (1997) describe three cases of people who exhibit psychotic phenomena, the experience of which actually helped them in times of crisis. They demonstrate how the only successful way of demarcating such benign cases from pathological psychosis is by reference to the values and beliefs of the individual - these being obviously culturally influenced factors. For further examples of culture's pervasive influence on phenomena, often seen as indicative of psychopathology, see: Larøi et al. (2014), NiaNia, Bush, and Epston (2016).
} 
explicating our own concept, we will therefore demarcate norms of relevance to mental disorder in a similar way.

In this paper we aim to take a similar position, and to develop it into a coherent and more comprehensive conception of mental disorder. This will largely consist of situating the functional view within a broader framework of human functioning. There are multiple reasons for doing so. For one, it stems from the likely complex and multi-scale causal structure of mental disorders (Kendler, Zachar, \& Craver, 2011). Situating conceptual work on mental disorder within an encompassing framework of human functioning will hopefully provide scaffolding for later integrative work. Furthermore, socio-cultural factors are not merely causally implicated in mental disorder, as is well known, but often play constitutional/definitional roles (Fulford, 1999; Fulford \& Jackson, 1997; Larøi et al., 2014; NiaNia et al., 2016). Integrating our understanding across these levels will therefore likely require a non-reductionistic and rich understanding of human functioning in general. One capable of valuing biological levels of explanation ${ }^{5}$ while simultaneously understanding culture and social embeddedness as more than an after-thought or as merely supervening on physiological processes (Kirmayer, 2006; Kirmayer \& Crafa, 2014). We agree with Kirmayer and Ramstead (2017) that the concepts of embodiment and enactment have huge potential in this regard, and therefore, in line with our previous work, we will be using $3 e$ cognition as such a framework (see; Nielsen \& Ward, 2018a).

Regarding the second requirement implied by Muders (2014) - that a concept of mental disorder be clear about what it takes its relevant norms to be - we will

\footnotetext{
${ }^{5}$ By biological we here mean physiological, molecular etc. However, Frisch (2016) argues convincingly that 'biological psychiatry' would be all the more biological with the inclusion of behavioural and environmental levels.
} 
return to this issue in the next section when we discuss the origins of normativity, links to embodied enactivism, and unpack further the concept of a functional norm. For the current time however, we would point out that this requirement points to the need for any successful concept of mental disorder to be situated within a broader framework of human functioning, one that provides the beginnings of purpose to the human condition. This is because in order to say that there are better and worse ways for individuals to act and be (i.e., for there to be normativity regarding human functioning), we need to establish what 'better' and 'worse' could possibly mean in a world of facts (Hume, 1978). To parallel this point more simply, it seems prima facie true that labeling something as dysfunctional should require comparison to an idea of what it means to function correctly. As we will show in later sections, 3e cognition thinking can provide a means of doing this.

\section{Origins of Natural Normativity and Links to 3e Cognition}

We will now shift gears and start laying the ground-work for our own normative concept of mental disorder. We first overview two very similar systems of thought regarding the natural origins of normativity that come from outside of $3 \mathrm{e}$ cognition; this will offer more clarity of what we mean by the concept of a functional or natural norm. We then introduce the field of $3 \mathrm{e}$ cognition and connect this idea of natural normativity to core ideas from the 3 e field. Finally, we argue that $3 e$ thinking allows for an extension of these ideas and that two key concepts highlighted throughout these areas can be used to demarcate mental disorder from the benign namely, self-maintenance and adaption.

\section{Functional/Natural Normativity}


The ideas explored in this section have been developed by Wayne Christensen and Mark Okrent in separate works on the origins of normativity (Christensen, 2012; Christensen \& Bickhard, 2002; Okrent, 2017). While these authors do not cite each other, they express remarkably similar ideas: that norms are inherent in selfmaintaining and adaptive systems such as life forms and arise in service to those systems continued adaptive functioning within their environment. More specifically, norms are seen as supporting the organizational autonomy ${ }^{6}$ of a system.

Organizationally autonomous systems on Christenson's view are those that “...possess a process organization that, in interaction with the environment, performs work to guide energy into the processes of the system itself.” (Christensen \& Bickhard, 2002, p. 3). In other words, they are thermo-dynamically open but selfmaintaining systems ${ }^{7}$. To use the example of life forms, organisms are very much in a far-from-equilibrium state when contrasted with the wider environment within which they are embedded; it's very easy for life forms to die, but hard for them to keep living. The persistence of an organism relies on a set of balanced conducive states and processes (self-maintenance), but also that these states and processes change in response to alterations in the environment in a way that serves selfmaintenance (adaption). These states and processes occur both within the individual (e.g. blood pressure and circulation), and within the environment (e.g. sufficient oxygen). These states and processes are the functional norms of the organism. Importantly for our purposes, behaviors of the whole system, so long as they serve the continued function of the organism, can also be seen as functional norms (e.g.

\footnotetext{
${ }^{6}$ We have added the descriptor 'organisational' to differentiate it from personal autonomy, a related but separate concept. For clarity we have, throughout this article, tended to refer to 'self-maintenance and/or adaption' so as not to introduce confusion with personal autonomy - valuation of which varies across cultures. ${ }^{7}$ The connection to 3e thinking is clear here, e.g. Varela, Thompson, and Rosch (2017), but links can also be made to Free Energy Principle theory; see Kirchhoff (2016).
} 
seeking food and shelter); “...for an entity to be alive is in part for it to interact with its surroundings in ways that are instrumental to its continuing life, given the kind of thing it is, from the 'standpoint' of the living thing there is a right and a wrong way to carry out that interaction.” (Okrent, 2017, p. 28). These accounts view “... normativity as inherent in the organization or form of living systems...” (Christensen, 2012, p. 104). This is why functional norms are often called natural norms.

The largest point of demarcation between these two authors is that Christensen is oriented to a systems perspective, and Okrent to one of organisms and agents. Both view norms as arising from the teleological purposiveness of selfmaintenance and adaptivity. For Okrent this is grounded in the nature of being an organism, and whether other kinds of things can give rise to such norms is an open question $^{8}$. Christensen is not bound to organisms as the only known sources of normativity in this way. Christensen's view makes it more explicit that ecosystems, social institutions, and other autonomous systems may conceivably have their own non-derivative functional norms (Christensen, 2012; Christensen \& Bickhard, 2002). For example, these norms might relate to levels of predation in an ecosystem, or availability of coffee in a busy office.

This overview hopefully elucidates what we mean by functional/natural norms. To further clarify however, we will briefly cover two types of norms that do not count as functional norms. Firstly, a functional norm is very different to norms based on typicality. Norms based on typicality are those that aren't functionally important and are simply based on deviation from the usual distribution - e.g. having a non-problematic benign growth or having purple hair; neither is typical, but

\footnotetext{
${ }^{8}$ Okrent does note that "Whether or not it is also the case that norms only arise in the context of life remains to be seen." (Okrent, 2017, p.28).
} 
neither is either a problem. These are often referred to as 'statistical' norms, and they are not seen as prescriptive (Okrent, 2017). As such, statistical norms cannot be of direct use for defining dysfunction or disorder, a point implicitly supported by Banner's (2013) construal and noted by Jefferson (2014) in the discussion earlier; "A statistical notion of dysfunction and pathology is too thin to be useful for medical practice." (Jefferson, 2014, p. 2).

Secondly, it is quite common in the literature to see norms of human functioning as derived from a component's apparent evolutionary function (Troisi \& McGuire, 2002; Wakefield, 1992). Norms based on purported evolutionary function are much more similar to the account at hand than statistical norms, in that they are prescriptive rather than merely statistical in nature. However, construing norms as natural based on their apparent evolutionary function faces a knowledge problem: we cannot know for certain that we have the evolutionary story correct, nor that other unknown functions aren't being simultaneously served by the state or process in question (Christensen \& Bickhard, 2002). Furthermore, such an account does not leave room for adaptive deviations from the evolutionary norm (Christensen \& Bickhard, 2002; Troisi \& McGuire, 2002). This is hugely problematic given the importance of adaptive phenotypic variation for evolutionary theory. As such, we do not see evolutionary theory as providing a rich enough account of human functioning to support an understanding of disorder, at least within the mental realm (however, for a good attempt at such a construal, see Troisi \& McGuire, 2002).

As a fictional example to flesh these differentiations between functional, statistical, and evolutionary norms we use the example of Jim. Jim has three arms, his third arm sits underneath his right. Importantly, Jim's third arm does not get in the way of his functioning, in fact Jim is better at many tasks than plain old two- 
armed people. Jim's arm therefore breaks norms of typicality (most people don't have three arms), and etiological/evolutionarily derived norms (we did not evolve to incorporate a third arm). However, Jim's third arm does not break the functional norms of 'Jim the complex autonomous system' because it does not get in the way of Jim's ability to meet his needs relevant to self-maintenance, nor impact his ability to adapt to environmental changes. On our view then, Jim should not be seen as disordered.

As a point of clarification, we are not saying here that the existence of functional norms cannot sometimes be inferred from statistical comparisons across individuals. Taking the example of blood pressure: we know what sorts of parameters are medically acceptable based on research studies, and that certain blood pressure thresholds are associated with harmful outcomes such as fainting, heart attacks, strokes, etc. This sort of inference seems reasonable, at least at the physiological scale where the states and processes that constitute functional norms are somewhat more stable across individuals, and deviations from norms often have more obvious effects (e.g. blood pressure is clearly definable and measurable, similar levels count as too high or too low across individuals, and deviation from the norm can result in outcomes that immediately challenge the self-maintenance of the individual). The inference from typicality and associated risk across the population to a normative claim about an individual's blood pressure therefore seems reasonable. For reasons we will return to later, whether the same sort of inference can be made when considering behaviors of an organism that do not seem to directly serve some obvious biological norm remains to be seen. We will argue that they cannot. Before doing so, we must first consider if views such as those of Christensen and Bickhard (2002), and Okrent (2017), can be extended to inform an understanding of the 
normativity of complex human behavior. We believe they can, and that the tools to do so are found within the field of $3 e$ cognition, which we will now describe.

\section{Overview of 3e Cognition}

In this section we aim to describe the $3 e$ Cognition perspective rather than argue for it. Our interest is in the implications of this approach for our understanding of psychopathology, rather than the philosophical justification for it (key literature supporting the $3 \mathrm{e}$ view is referenced at the end of this section).

By 3 C Cognition we refer to the view that the mind is fully material, and that it is constituted by not just the brain, but the brain-body system; we are embodied beings. More than this, interactions with the physical and social environments within which the organism is embedded are seen as necessary conditions for the development of the mind over time; we are embedded. We are also enactive creatures (Gallagher, 2017; Thompson, 2007). According to enactivism, the mind is not a thing above and beyond the organism in the Cartesian sense. Rather it is an interrelated set of capacities that are essentially dispositions to act in accordance with an organism's needs, interests, and respective goals (note that these would count as functional norms as described above). Mental processes are necessarily embodied in the brain, nervous system, and all other biological systems of the body. Phenomenological experience emerges (is enacted) by virtue of the organism making sense of and adapting to the world (Di Paolo, 2005); it is the body experiencing itself and the world (Fuchs, 2017). The enactive/embodied conception of human functioning is based on a relatively simple idea: human psychological functioning and sense of meaning is shaped in fundamental ways by bodily experience and the needs of the body as a biological organism within its environment (Gallagher, 2006; Thompson, 2007; Varela et al., 2017). It is not possible for minds to function 
independently of the body (Thompson \& Cosmelli, 2011); they are essentially interrelated sets of processes.

Thus the mind can be seen, not as a linear symbol processing machine with a defined inputs and outputs, but as an emergent property of the whole organism arising from interactions in the brain-body-environment system to better serve its self-maintenance and adaption (Thompson, 2007). Human cognition then is much less dependent on cognitive representation and processing: "a natural cognitive agent - an organism, animal or person - does not process information in a contextindependent sense. Rather it brings forth or enacts meaning in structural coupling with its environment." (Thompson, 2007, p. 58). This also has the effect that, from the perspective of $3 e$ Cognition, the affective nature of our experience - the meaning that is immediately apparent in the world around us or what Maiese (2016) calls affective framing - can be seen as real (Colombetti, 2014; Colombetti \& Thompson, 2008). We have here simply offered an outline of 3 e Cognition, for a defense of the 3e viewpoint see: Colombetti, (2014); Durt, Fuchs, and Tewes, (2017); Fuchs, (2017); Gallagher, (2017); Gibbs, (2005); Maiese, (2016); Thompson,( 2007); Hutto and Myin (2012, 2017) and Varela et al., (2017).

Readers familiar with these ideas will note that we are using the term $3 \mathrm{e}$, when often the term $4 \mathrm{e}$ is used. We do so because we do not subscribe to the fourth 'e' - extension (where the mind is seen as partially constituted by the external environment; Clark \& Chalmers, 1998). Our reasons for this are multiple but we will briefly allude to them. Firstly, we do not see full extension as compatible with enactivism and embodiment given that the latter two emphasize the process of continual separation between organism and environment (self-maintenance), while extension de-emphasizes this (Maiese, 2017). Secondly, enactivism holds that 
meaning is always relational - it is generated by an organism through its needful relation with the world (Thompson, 2007). The constitutional boundaries of the organism become blurry and ever-changing under extension (Maiese, 2017), and this seems to make the nature of the enactive relation very unclear. Thirdly, for our purposes at least, subscription to embeddedness (rich and necessary causal relations between organism and environment), as opposed to extension (constitutional expansion), can achieve much of the same conceptual ends while allowing for clearer explanations, e.g. it would be very hard to explain the depression of some client 'John' if we spend our time trying to decide where 'John' ended and his environment began. Fourthly, many brands of extension seem to rely on an informationprocessing account that we disagree with due to their running afoul of the hard problem of content (for more on this see: Harvey, 2015; Hutto \& Myin, 2012). Finally, Thompson and Stapleton (2009) show that once the concept of extension is cut to size in-order to fit with embodied enactivism then genuine extension of the mind becomes a much less remarkable and quite rare phenomenon.

\section{The Deep Continuity Thesis, Cultural Embedment, and Normativity}

The idea of naturally derived normativity explored earlier in this section is remarkably similar to a key set of concepts in $3 \mathrm{e}$ Cognition. In particular, there is great similarity here to the deep continuity thesis (DCT). This is the idea that that the origins of mind arise from the same process structures that support and define life (Kirchhoff \& Froese, 2017; Thompson, 2007). Under the DCT, meaning arises from an organism's needful relation with its environment; to self-maintain requires the acquisition of energy from the world and avoidance of threats to the self (Thompson, 2007). At the cellular level this process is referred to as autopoiesis (Thompson, 2011; Thompson \& Stapleton, 2009). This needs-based relationship changes the 
environment to one of meaning and valence for the organism, making the mind and our relation to the world inescapably affective in nature ${ }^{9}$, whilst still thoroughly embodied (Colombetti, 2014; Colombetti \& Thompson, 2008; Maiese, 2016). This does not mean that basic life forms, or plants, are conscious in a self-aware or reflective sense (this would be seen to come later, with the evolutionary development of a nervous system or some equivalent). Rather, according to the DCT, all life forms are viewed as having a non-conscious subjectivity or 'zero-point', and a nonconscious embodied 'concern' (i.e. a self-perpetuating structure) for the continuation of the self (self-maintenance) in the face of changing and precarious environmental conditions (adaption) (Di Paolo, 2005; Thompson, 2007; Thompson \& Stapleton, 2009). Insofar as an organism should act to maintain its own life, there are states, actions, and processes that the organism should be in or perform. These states, actions, and processes change in accordance with the current needs of the organism and the constraint of the environment. Consistent with Christensen and Okrent's work, the DCT places the origins of normativity and meaning in the self-maintenance of organizationally autonomous and adaptive complex systems - namely, life forms (Christensen, 2012; Christensen \& Bickhard, 2002; Okrent, 2017).

3e thinking however, offers an extension of this account of normativity. Two key 3e concepts are important here. Firstly, that of embedment described earlier, where interactions with the environment are necessary for the development of the mind. This refers to both a physical and, especially in humans, a socio-cultural environment. The second key idea relevant to our purposes here is the constitutional view of culture (CVC). Most succinctly espoused in the introduction of Durt et al.

\footnotetext{
${ }^{9}$ It is fascinating to note that Okrent (2017) also arguably touches on this point. In chapter 2 he states, "for an organism to perceive its world is to perceive what is instrumentally important to the organism..." (p.31). Thus, he ties perception to the enaction of meaning via the pragmatic needs of a living organism, in a very similar way to the $3 e$ authors cited here.
} 
(2017), CVC is in many ways an elaboration of embedment. According to the CVC, groups of individuals and the artifacts they produce constitute a cultural ontology; a collaboratively generated shared world of significance and meaning that facilitates intra-group behavior and the transmission of tools, knowledge, and ways of knowing (Durt et al., 2017; Kirmayer \& Ramstead, 2017). This shared world, or habitus (in the sociological sense), is embodied within the habits and practices of the group which are passed on to and developed by younger generations because they represent adaptive ways of understanding, managing, and altering the environment (Henrich, 2015; Heyes, 2018). Interestingly, such a perspective can even be shown to encompass so-called higher level cognitive practices such as mathematics and reasoning about the minds of others (Gallagher, 2017; Heyes, 2018). Significantly, this shared world, while being co-generated by the group, also represents a major reshaping of the environment within which individuals reside, thereby constructing the ontogenetic and phylogenetic development of individuals in ways that the group has found to be adaptive (Durt et al., 2017).

These two ideas in combination have allowed authors such as Maiese (2016) and Di Paolo (2005) to describe how, in conceptually and socially sophisticated animals such as ourselves, more complex tendencies in behavior can develop, embodied within the dynamics of the organism system. Building upward from the enactive core of meaning rooted in the needful relation between organism and environment, Maiese and Di Paolo demonstrate how irreducible higher-order socioculturally mediated values can emerge. Over evolutionary and life-span time scales, these behavioral/valuation-al tendencies are selected for and developed, as they allow the organism to flourish in accordance with the constraints of the sociocultural environment (which they as a group constitute and as individuals reside within). These behaviors are therefore irreducible functional norms, serving the 
flourishing and by extension the self-maintenance and adaption of the organism system within the socio-cultural environment. As individuals and cultures then, humans generate their own values/meaning. Within our framework, we have labelled these interpersonal prudential norms ${ }^{10}$; examples may include mastery, personal autonomy, and social connectedness (Nielsen \& Ward, 2018a). Functional norms then, as used within our framework, are not simply inherent in those biological states, processes, and basic behaviors of the organism that immediately support them (e.g. seeking food and shelter), but are also evident in more complex behaviors that indirectly serve the continued functioning and maintenance of the organism via reciprocal relations with the socio-cultural environment. Maiese (2016) offers the example of being a good driver: we wish to be good drivers not simply so that we can avoid crashing, but to demonstrate our mastery which has positive social implications for us.

While biological norms are similar across individuals, interpersonal prudential norms vary in the degree to which they are endorsed across cultures. This is because culture constitutes a significant variation in the environment, thereby placing differing constraints on how individuals can best achieve their needs. Endorsement will also vary across individuals due to dispositional differences (whether learnt or genetic). This has implications for the process by which we can gain knowledge of norms. As discussed earlier regarding the norms of bodily processes such as blood pressure, the inference from typicality and associated risk at a population level to a normative claim about an individual seems reasonable. However, things get murkier when we shift to functional norms of behavior. For example, the degree of personal autonomy required to support functioning will vary

\footnotetext{
${ }^{10}$ In our previous paper we referred to these as values rather than norms, we have shifted to the use of norms for the sake of clarity (Nielsen \& Ward, 2018a).
} 
across contexts, cultures, and individuals. The inference from typicality to functional norm is a lot more tenuous within the domain of behavior than it is when considering physiology or the like (Fulford, 2002). This is because there are many different ways for individuals, groups, cultures, societies, and ecosystems to meet the needs required for their self-maintenance. In other words, these higher-level systems have a larger set of functional states. In contrast, the human circulatory system and other such internal bodily systems, have a much smaller set of functional states - e.g. not much needs to change about the circulatory system to result in the death of an organism. In practice, this means that a clinical psychologist or psychiatrist will always be asking the question "is this a problem for this individual within their context?” Whereas, for a medical doctor, answers to this question will be easier to arrive at.

\section{What Counts as Mental Disorder?}

Drawing together this ground-work and weaving it in with the previous discussion on normativity in the concept of mental disorder, a view emerges similar to but more developed than that argued for by Banner (2013) (also see Frisch, 2016). What counts as mentally dysfunctional is any set of behaviors (inclusive of cognition, perception - anything the organism does) performed by an organism that significantly violates its own functional norms, in that it is acting counter to its own self-maintenance and adaption needs ${ }^{11}$. The persistence of this pattern of behavior

\footnotetext{
${ }^{11}$ We use these two processes within this definition because, under a 3e conception of human functioning these are fundamental processes. Other values/functional norms should be considered when demarcating dysfunction, such as the interpersonal prudential norms mentioned earlier. However, we believe that a reasonable link needs to be made back to these fundamental processes if a diagnostic label is going to be ethically applied. To not demonstrate such a link risks pathologizing individual or cultural variances in modes of functioning.
} 
thereby threatens the organism's organizational autonomy and as such, should be considered disordered ${ }^{12}$.

This is similar to Banner's construal in that it is functionally defined, thereby positioning it beyond the false dichotomy of weak versus strong evaluativism. As argued earlier, this is a significant strength as the act of diagnosis is then justifiable by reference to individuals and their needs; staving off Szaszian claims (unlike strong evaluativism), while also not ignoring how culture shapes many of those needs in the first place (as per weak evaluativism). However, being situated within the broader framework of 3 e cognition offers advantages over Banner's functionalism. This framework brings greater conceptual specificity, provides justification for the use of functionality as the crux of the definition, encourages ecological considerations including socio/cultural elements, and offers a rich and coherent system for conceptualizing relevant factors such as mind and culture. We will now continue to develop this construal, first by highlighting some key strengths, and then by exploring a foreseeable counter-argument to which we reply.

\section{Evaluating this Position}

We think that a strength of this framework is that it is in many ways congruent with a medical understanding of physical illness, while also highlighting the differences between the bio-medical and psychological domains and their respective conceptual needs. A significant violation of the functional norms of an organism system at a biological scale essentially constitutes an injury or medical condition. Similarly, on our view a significant and continued violation of functional norms of the organism system at a behavioral or psychological scale is a

\footnotetext{
12 Note here that we draw a distinction between 'dysfunctional' and 'disordered', with disordered referring to the persistence of significantly dysfunctional behaviour.
} 
psychological disorder. As explored above, one key difference between these domains is that in the former it is generally safer to infer the existence of a functional norm from a statistical one. Functionality of behavior and psychology is, in contrast, diverse in that there are many ways to be functional - as exampled by cultural variation (Fulford, 2002). It is therefore ethically questionable to infer that a norm derived from typicality is a functional one within the psychological domain, because whether it counts as a functional norm is going to be much more individually and contextually specific. This framework therefore prescribes great attention to the role of the context in shaping an individual's way of functioning.

At all scales of analysis, the 3e framework highlights that an organism is attempting to act in accordance with its inherent purpose - to adapt (Di Paolo, 2005) and self-maintain (Thompson, 2007). Just as getting a cold reflects faltering of the immune system to adapt to the challenge of a pathogen, mental or behavioral disorders often reflects a faltering of the organism attempting to adapt to the challenge of a changing environment ${ }^{13}$. 'Faltering' is here used because outright failure is inappropriate; the organism is still alive. An example of this would be a child growing up in a difficult family context where cycles of coercion have negatively reinforced his escalating of aggressive behavior (Granic \& Patterson, 2006; Smith et al., 2014). We know this will not serve the child well in other contexts, and may disrupt other norms of development (Erskine et al., 2016). However, the aggression has developed due to the constraints of the family system and the child's adaption to this environment. A further example would be a refugee from a war zone whose previously adaptive bias towards interpreting others' actions as aggressive is now

\footnotetext{
${ }^{13}$ We realise this is not a perfect analogy - many symptoms of a cold may actually be seen as a functional and typical response to the presence of the pathogen.
} 
dysfunctional within his present, largely peaceful, context. Both examples highlight the need for consideration of context over time rather than just the role of the current environment. The 3e perspective allows for, and indeed encourages, recognition of both of these sides; that this behavioral pattern is an adaption to the environment, but that it is also very likely to be maladaptive in other contexts and is maintaining a family dynamic that is problematic for both other family members and the continuing development of the child. Our framework then, encourages the consideration of context. The question being: in what way is the behavior attempting to serve the person's needs within their context (past or present), and are there other ways for these needs to be met that would represent a more balanced normative equation?

This brings us to what we see as a further strength of thinking about disorder in this way. An individual's functional norms do not necessarily all point to a single prescribed action (and if they do, these tend to be areas in our lives in which decisions as to which action to take are clear and easy). Instead, functional norms often compete, and compromise is required. For example, it's ideal to sleep 6-8 hours a night, but sometimes we have some approaching deadline and need to compromise on this; staying up late to finish some important project. One can act in accordance with one norm, while violating another. When it comes to norms, compromise is the norm! If however, I stay up late to complete work regularly, perhaps for less and less important projects and resulting in chronic tiredness, then the normative equation begins to look unbalanced. In other words, this pattern of behavior starts to look dysfunctional.

This idea of an unbalanced normative equation is worth fleshing out with a clinical example. Imagine a client where some behavior (e.g., cutting) is serving some 
function (e.g., emotional regulation). To use normative language, the cutting is serving the norm of emotional stability. However, in the process, other norms are adversely impacted (e.g., having unbroken skin). Two elements are of importance here. Firstly, while the cutting is serving a norm/function, this does not mean that it is on the whole 'functional'. Other norms are being violated by this action (having unbroken skin), and there is risk of breaking even more vital norms (e.g., being infection free, undamaged arteries/veins). It is this element that is important when considering whether the equation is reasonably balanced or not; whether the pattern of behavior and its consequences are on the whole functional (ranging from ideal to roughly functional) or dysfunctional (the individual's functional norms are being or are at significant risk of being significantly impacted). The second element to consider in this example is whether there are clearly ways in which the function performed by the cutting behavior may be achieved in a significantly less normatively imbalanced way (e.g., emotional regulation strategies). Insofar as there is a less negatively impactful way to achieve some norm, and that the compromising of other collateral norms is significant, we are justified in offering assistance. When the functional norm breaking behavior takes a recognized causal and constitutional form, labeling with a diagnosis to facilitate communication and treatment across organizations is our society's way of achieving and providing this assistance.

\section{A Possible Objection}

Many readers at this point will be concerned that we have ignored an obvious counter example. This would be a situation where the social context is placing unjustified constraints on someone, and where defiance of these constraints appears somewhat 'dysfunctional'. Examples would include acts of rebellion in a totalitarian society, and gay people expressing their sexuality in a homophobic society. At first 
glance it may seem that according to our view these are instances of mental disorder because both acts are not adaptive within the social context given the risk they bring to the individual. This is obviously a problematic conclusion. This issue seems to underlie the intuitive need for some sort of recognized dysfunction or lesion alongside the normatively defined 'harm', as in the harmful dysfunction analysis (Wakefield, 1992, 1997). The intuition seems to be that this requirement allows for an easy response to such counter examples; the ontic distinction from typicality at some sub-personal level makes the disorder seem more 'real'. However, we will argue that, with an addendum justified by the broader $3 \mathrm{e}$ framework, our functional construal can exclude such cases. It is therefore more parsimonious than two-part models, and does not unduly privilege the sub-personal. First however we must explore the issue in a little more depth.

In general terms the violation of norms of the socio-cultural systems (functional, legal, civil, or otherwise), do not represent mental disorder under our framework (they may however represent a crime, immoral act, or social faux pas). Rather we are specifically concerned with the functional norms of the individual. This is what separates our claim regarding the normativity of mental disorder from the Szaszian view, under which disorders are defined by the violation of sociocultural norms (and are therefore not justifiable if the labeling of disorder is truly intended to be in the interest of the individual; Szasz, 1960, 1963, 1974).

Unfortunately, things are rarely this simple. Under the CVC, one may note that there is a complex two-way relationship between the norms of an individual and the norms of a culture or society. While the norms of the culture serve the continued survival and functioning of the collective, the collective itself is of course constituted by the individuals and therefore the functional norms of the culture will, largely and 
for the most part, serve the majority of the individuals' survival. Going in the other direction, the norms of the group are a dominant constraint on how the developing individuals within that group context learn to function. Large parts of the intradependent set of functional norms operating on an individual are therefore shaped by their cultural context across development. Someone who grew up in urban Japan will have a different way of functioning than someone who grew up in bible-belt USA, and so on. If culture is the ways of knowing, being in, functioning, and making sense of the world, shared across a particular group (Durt et al., 2017), then consideration of culture when asking normative questions is always going to be relevant.

This means that a discussion of individual normativity must explore the role of culture but, more practically, also makes teasing apart the functional norms of an individual from the norms of the culture in which they reside challenging. This is especially true when someone is part of a cultural minority or of a culture that is less recognized in the mainstream, as such individuals are effectively living between two worlds and exposed to contrasting ways of functioning. One particularly interesting example, that highlights the importance of interplay of individuals and culture in shaping the functionality or disorder of a behavior, is how experiences that from a western viewpoint would certainly be classified as hallucinations are interpreted much less pathologically in many cultures. For individuals embedded within such cultures, the consequences for their functioning are much less severe, sometimes even positive (Fulford \& Jackson, 1997; Larøi et al., 2014; NiaNia et al., 2016).

This gets us to the problem. In recognizing that social context is a huge part of the individual's environment, socio-cultural norms can sometimes be imported as derivatively functional for the individual. It therefore seems that such cases as rebellion in totalitarian society, or expression of queer sexuality in a homophobic 
society, must be counted as disordered under a functional construal. However, the $3 \mathrm{e}$ orientation of our framework can help us in navigating this situation. 3e thinking places the anchor point of consideration at the level of the individual; as the experiencing agent, for which meaning exists. In light of this, it seems very odd to refer to a norm as functional for an individual if it stems from a socio-cultural norm that does no work for, or in fact is running counter to, the self-maintenance and adaption of the individual in question. We therefore suggest the following addendum that helps clarify why such examples do not count as disorder under our framework:

A norm, even if apparently functional, should not be used to define disorder if it is derived from (secondary to) either:

a) A non-functional norm of a higher-order system, or

b) A functional yet arbitrary norm of a higher-order system that is impinging on the self-maintenance and/or adaption of the lower order entity.

We will now explain and justify this addendum through the exploration of the problematic cases. Firstly, the expression of homosexual orientation in a homophobic society. As explored above, an argument could be made that this not functional for the individual because it risks persecution. However, the socio-cultural norm of homophobia is a statistical/religious/erroneous moral norm, not a functional one. We now know that allowing honest expression of sexuality with our societies does not result in societal collapse. Therefore, the constraint placed on the individual by the homophobic norm is not justified; the problem is with society and with its norms not working for the individual, not with the individual themselves ${ }^{14}$.

\footnotetext{
${ }^{14}$ As a parallel point it is also very difficult to see within this example how a norm that is so constraining on the autonomy of the individual can really be said to be 'functional' for that individual.
} 
Accordingly, the addendum above specifies that while it may, in a homophobic context, be somewhat functional to hide one's sexuality, continued expressions against this particular functional norm should not be seen to constitute mental disorder. This is because the dysfunctionality of honest expression of one's sexuality is derived from a non-functional socio-cultural norm. Insofar as, from a CVC view, it is society's role to serve its constituents, the dysfunction is with the homophobic society, not with the homosexual individual.

Secondly, concerning rebellion and other risky political acts. Once again, an argument can be made for such behaviors being dysfunctional because they risk the self-maintenance and/or adaption of the individual. This is a slightly more complicated situation because, despite moral qualms, it may be argued that the overly restrictive norms of a totalitarian society are functional in that they are helping to maintain the stability of the society in question ${ }^{15}$. However, there is a sense in which the functionality of such norms is arbitrary; we know that other societies exist that do not rely on totalitarian norms for their continuation. Assuming again that the purpose of a society is to serve its constituents (as per the CVC), the fact that this society is impinging on its member's self-maintenance and/or adaption to survive suggests that the dysfunction is at a societal level, not with the rebellious individual (and indeed this seems to go some way in justifying their action for change). In accordance with this reasoning, the above addendum rules out basing the labeling of mental disorder on seemingly functional norms derived from functional yet arbitrary socio-cultural norms.

\footnotetext{
${ }^{15}$ Once again, it is difficult to see how such overly restrictive norms are in any true sense 'functional' for the individuals being constrained. However, given the context it becomes in a sense 'functional' to abide by it.
} 
Having questioned societal norms in light of individual norms, it is interesting to question individual norms in light of the social ${ }^{16}$. There are certainly cases where the social trumps the individual ${ }^{17}$. Even when some action is functional for the individual, should it cross certain social norms then this would seem to constitute a social faux pas, crime, or immoral act. The framework presented here does not excuse such actions (although we would hope it would encourage compassion in seeking to explain them). In cases where patterns of such action become a learned way of functioning for an individual though, two interesting categories seem to emerge. The simplest of these is non-pathological; those that achieve their own selfmaintenance and adaption in disregard of social/legal/moral norms. This category would range from selfish people to career criminals. The second case is more interesting for our discussion here; those whose patterns of social norm violations actually work against their own self-maintenance and adaption within their social environment, and are therefore pathological in the sense defined here - i.e. personality disorders. Under our framework personality disorders do seem to count as disorder, but the harm to the individual is mediated by the breaking of social norms rather than by the crossing of individual norms directly. These constructs then are different in kind to both “regular” psychopathology where individual

\footnotetext{
${ }^{16}$ Thank you to one of the reviewers for suggesting this dialectical approach.

${ }^{17}$ When an individual acts in the interest of the group, in contradiction to their own interests, then there may be a concern that our framework labels such a behavior dysfunctional, and its persistence disordered - some sort of 'altruistic personality disorder' if you will. This is an issue that needs further thought, but our intuition is that our framework is not individualistically biased in this way. Within the timeframe of the act, altruistic behaviors seem to reflect an emphasis on individual and biologically immediate norms relative to socioculturally generated norms. However, because these norms benefit the individual at other times, then as per to our addendum, these altruistic norms should not be used to define disfunction. Thus, someone may, to a certain degree, act against their own self-maintenance and adaption in a non-dysfunctional way. The limiting factor is that, largely and for the most part, the norms they are following during the act benefit them at other times.
} 
functional norms are directly impinged, and social deviancy where social norms are directly violated.

Before closing this section, it is worthwhile to briefly consider some current personality disorders as they represent complex normative cases. On the current construal some personality disorders end up looking more valid than others. For example, it is hard to imagine a group that functions well and serves the interest of the constituent members where interpersonal styles akin to those seen in narcissistic personality disorder, borderline personality disorder, or anti-social personality disorder are encouraged. The socio-cultural norms in question then, seem both functional and non-arbitrary, with the outcome being problematic interpersonal functioning (although in the case of narcissism there would be a genuine argument to be made that the problems that arise primarily concern others rather than the individual being diagnosed). In other cases, however, such as in schizotypal personality disorder, the socio-cultural norms being broken seem very much to be predominantly statistical, making this a very questionable diagnostic category under the current framework. Finally, schizoid personality is very interesting to consider while statistical norms are certainly being broken in such cases, it is hard to understand how this disorder represents a functional problem for the individual concerned. This 'disorder' therefore seems more likely to be simply a different mode of functioning.

\section{Conclusions and Summary}

We have argued elsewhere that, ontologically speaking, all forms of psychopathology are likely constitutionally and causally complex phenomena, situated across multiple scales of analysis. Despite their complexity they are the sorts of 'natural' things we can seek to explain, and when they occur with some reliability 
they seem like good candidates for being natural kinds of a type-causal variety (Nielsen \& Ward, 2018a).

Ultimately though, a diagnosis is a claim that something is wrong with a person's functioning. A diagnosis is therefore a normative claim. Overviewing a brief sample of literature in this area, the most pertinent question seemed to be: which norms are relevant when demarcating disordered from benign conditions? Stier (2013) described current practice as including socio-cultural norms within this distinction, while Jefferson (2014) suggested this is unjustified and risks unsustainable relativism. We suggested that the most viable move was exemplified by Banner (2013), who starts to move beyond strong versus weak evaluativism; instead defining disorder by the functionality of behavior. In accordance with the requirements of a satisfactory mental disorder concept implied by Muders (2014), we have here attempted to develop this functional view into a fully-fledged and coherent position within this debate that makes clear what norms are at issue and where they are seen to come from.

Reconciling this functional view with science's naturalized view of the universe required an account of how purposiveness and normativity can arise, in order for there to be purpose and norms against which functioning is contrasted. We argued that 3 e cognition offers such an account which we have here explored, alongside consilient views of normativity, whereby norms arise in life-forms due to their organismic self-maintaining process structure and their adaption to the constraints of their environment (Christensen, 2012; Christensen \& Bickhard, 2002; Okrent, 2017; Thompson, 2007). From this position, mental disorder is a pattern of behavior (inclusive of all actions of the organism, such as thought and perception) that runs counter to its functional norms to a significant or atypical degree (Nielsen 
\& Ward, 2018a). Functional norms are those norms that support the organisms continued self-maintenance and adaption, and by extension, their ability to fare well in their communities (Di Paolo, 2005; Maiese, 2016). What exactly it means to 'fare well' for any individual will subtly change as a function of the individual, and will covary with the culture in which they learned to function. This welcomes intersection with cross-cultural psychology and psychiatry (Kirmayer \& Crafa, 2014; Kirmayer \& Ramstead, 2017).

Teasing apart the norms that serve the individual from those that serve the group is a complicated exercise. We have here argued that this distinction must rest on whether the norms of society are working for the individual, or put more technically, whether the norms in question support the individual's self-maintenance and adaption. A 3 e orientation therefore prescribes strong consideration of context and culture over time, while also focusing on the individual and their needs. A $3 e$ perspective on mental disorder, in that it subscribes to embedment, must recognize the role of culture in shaping the way that an individual functions. The functionality of a behavior, even those which we may dismiss as inherently pathological from a western context, is often contingent on the social environment, as well as the culturally informed manner of functioning and definition of 'flourishing' that the individual subscribes to. The 3e perspective encourages us to consider such rich variation and, through its basis in the organism's strive to survive as a basic predicate of all life, provides a basis on which to begin to tease apart the disordered from the functional at the level of the individual. We have further specified that, within this framework, functional norms of individuals that are derived from non-functional or arbitrary socio-cultural norms should not play a role in demarcating disorder. 
Despite their apparent functionality, such norms seem to represent a disorder of society rather than disorder of the individual.

Understanding the normative nature of diagnosis is vital for the purposes of being able to ethically justify our practices as psychologists, psychiatrists, and councilors. We believe that a 3 e conception holds potential in this regard given its ability to bridge the natural and the normative, and hope that our discussions here represent a step towards developing this perspective. Moving forward we intend to explore the implications of the framework presented here for the tasks of explanation and classification of psychopathology (Nielsen \& Ward, 2018b). As an upshot of the normative focus that the 3 e position brings, we must question the nature of the norms imposed by society. Institutions such as psychiatry and clinical psychology in being the arbiters of such strong normative labels as diagnoses are, and advocates for those in or in need of our care - have a responsibility to be critical of the norms of society when they touch on our domain of expertise. Importantly, this includes reflecting on our own institutional and personal norms of practice.

\section{Acknowledgements}

The Authors would like to thank Hannah Hawkins-Elder, Samuel Clack, Snita Ahir-Knight, and the rest of the EPC Lab at Victoria for their feedback during the writing of this article. We also appreciate helpful comments and support from Emma Ashcroft.

\section{Declaration of Conflicting Interests}

The Authors declare that there is no conflict of interest.

\section{Funding}


This research received no specific grant from any funding agency in the public, commercial, or not-for-profit sectors. 


\section{References}

Abouelleil, M., \& Bingham, R. (2014). Can psychiatry distinguish social deviance from mental disorder? Philosophy, Psychiatry, \& Psychology, 21(3), 243-255.

Banner, N. F. (2013). Mental disorders are not brain disorders. Journal of Evaluation in Clinical Practice, 19(3), 509-513.

Bolton, D. (2008). What is mental disorder?: an essay in philosophy, science, and values: Oxford University Press.

Browne, T. K. (2017). A Role for Philosophers, Sociologists and Bioethicists in Revising the DSM: A Philosophical Case Conference. Philosophy, Psychiatry, \& Psychology, 24(3), 187-201.

Christensen, W. D. (2012). Natural sources of normativity. Studies in History and Philosophy of Science Part C: Studies in History and Philosophy of Biological and Biomedical Sciences, 43(1), 104-112.

Christensen, W. D., \& Bickhard, M. H. (2002). The process dynamics of normative function. The Monist, 85(1), 3-28.

Colombetti, G. (2014). The feeling body. Cambridge (Mass.).

Colombetti, G., \& Thompson, E. (2008). The feeling body: Towards an enactive approach to emotion.

Deci, E. L. and Ryan, R. M. (2000). The "what" and "why" of goal pursuits: Human needs and the self determination of behavior. Psychological Inquiry, 11, 227268.

Di Paolo, E. A. (2005). Autopoiesis, Adaptivity, Teleology, Agency. Phenomenology and the Cognitive Sciences, 4(4), 429-452. doi:10.1007/s11097-005-9002-y

Durt, C., Fuchs, T., \& Tewes, C. (2017). Embodiment, Enaction, and Culture: Investigating the Constitution of the Shared World: MIT Press. 
Erskine, H. E., Norman, R. E., Ferrari, A. J., Chan, G. C., Copeland, W. E., Whiteford, H. A., \& Scott, J. G. (2016). Long-term outcomes of attentiondeficit/hyperactivity disorder and conduct disorder: a systematic review and meta-analysis. Journal of the American Academy of Child and Adolescent Psychiatry, 55(10), 841-850.

Frisch, S. (2014). How cognitive neuroscience could be more biological-and what it might learn from clinical neuropsychology. Frontiers in Human Neuroscience, 8, 541.

Frisch, S. (2016). Are mental disorders brain diseases, and what does this mean? A clinical-neuropsychological perspective. Psychopathology, 49(3), 135-142.

Fuchs, T. (2017). Ecology of the Brain: The phenomenology and biology of the embodied mind: Oxford University Press.

Fulford, K. (1999). Nine variations and a coda on the theme of an evolutionary definition of dysfunction.

Fulford, K. (2001). 'What is (mental) disease?': an open letter to Christopher Boorse. Journal of Medical Ethics, 27(2), 80-85.

Fulford, K. (2002). Values in psychiatric diagnosis: executive summary of a report to the chair of the ICD-12/DSM-VI Coordination Task Force (Dateline 2010). Psychopathology, 35(2-3), 132-138.

Fulford, K., \& Jackson, M. (1997). Spiritual experience and psychopathology. Philosophy, Psychiatry, \& Psychology, 4(1), 41-65.

Gallagher, S. (2006). How the body shapes the mind: Clarendon Press. Gallagher, S. (2017). Enactivist Interventions: Rethinking the Mind: Oxford University Press.

Gibbs, J. R. W. (2005). Embodiment and cognitive science: Cambridge University Press. 
Granic, I., \& Patterson, G. R. (2006). Toward a comprehensive model of antisocial development: A dynamic systems approach. Psychological Review, 113(1), 101.

Harvey, M. I. (2015). Content in languaging: why radical enactivism is incompatible with representational theories of language. Language Sciences, 48, 90-129.

Henrich, J. (2015). The secret of our success: how culture is driving human evolution, domesticating our species, and making us smarter: Princeton University Press.

Heyes, C. (2018). Cognitive gadgets: the cultural evolution of thinking: Harvard University Press.

Hucklenbroich, P. (2014). Medical criteria of pathologicity and their role in scientific psychiatry-comments on the articles of Henrik Walter and Marco Stier. Frontiers in Psychology, 5, 128.

Hume, D. (1978). A treatise of human nature [1739]. British Moralists, 1650-1800.

Hutto, D. D., \& Myin, E. (2012). Radicalizing enactivism: Basic minds without content. MIT Press.

Hutto, D. D., \& Myin, E. (2017). Evolving enactivism: Basic minds meet content. MIT Press.

Jefferson, A. (2014). Mental disorders, brain disorders and values. Frontiers in Psychology, 5, 130.

Kendler, K. (2016). The nature of psychiatric disorders. World Psychiatry, 15(1), 512.

Kendler, K., Zachar, P., \& Craver, C. (2011). What kinds of things are psychiatric disorders? Psychological Medicine, 41(6), 1143-1150.

Kirchhoff, M. D. (2016). Autopoiesis, free energy, and the life-mind continuity thesis. Synthese, 1-22. 
Kirchhoff, M. D., \& Froese, T. (2017). Where there is life there is mind: In support of a strong life-mind continuity thesis. Entropy, 19(4), 169.

Kirmayer, L. J. (2006). Beyond the 'new cross-cultural psychiatry': Cultural biology, discursive psychology and the ironies of globalization. Transcultural Psychiatry, 43(1), 126-144.

Kirmayer, L. J., \& Crafa, D. (2014). What kind of science for psychiatry? Frontiers in Human Neuroscience, 8, 435.

Kirmayer, L. J., \& Ramstead, M. J. (2017). Embodiment and Enactment in Cultural Psychiatry. In C. Durt, T. Fuchs, \& C. Tewes (Eds.), Embodiment, Enaction, and Culture: Investigating the Constitution of the Shared World (pp. 397): MIT Press.

Larøi, F., Luhrmann, T. M., Bell, V., Christian Jr, W. A., Deshpande, S., Fernyhough, C., ... Woods, A. (2014). Culture and hallucinations: overview and future directions. Schizophrenia Bulletin, 4O(Suppl_4), S213-S220.

Lieberman, J. A., \& Ogas, O. (2015). Shrinks: The untold story of psychiatry: Hachette UK.

Maiese, M. (2016). Embodied Selves and Divided Minds: Oxford University Press. Muders, S. (2014). On the concept of the normative in the assessment of mental disorder. Frontiers in Psychology, 5, 129.

NiaNia, W., Bush, A., \& Epston, D. (2016). Collaborative and Indigenous Mental Health Therapy: Tātaihono-Stories of Māori Healing and Psychiatry. New York: Taylor \& Francis.

Nielsen, K., \& Ward, T. (2018a). Towards a New Conceptual Framework for Psychopathology: Embodiment, Enactivism and Embedment. Theory \& Psychology. Advance online publication. 
Nielsen, K., \& Ward, T. (2018b). What Are the Best Targets of Explanation in Psychopathology? A 3e Informed Proposal and Meta-Methodological Implications. Manuscript in preparation.

O'Connor, B. (2017). Mental Disorder as a Practical Psychiatric Kind. Philosophy, Psychiatry, \& Psychology, 24(4), E-1-E-13.

Okrent, M. (2017). Nature and Normativity: Biology, Teleology, and Meaning: Routledge.

Porter, D. (2010). Paradigms, consequesnces and the normative dimension. Association for the Advancement of Philosophy and Psychiatry Bulletin, 17, $12-15$

Sadler, J. Z. (2005). Values and psychiatric diagnosis (Vol. 2): Oxford University Press.

Smith, J. D., Dishion, T. J., Shaw, D. S., Wilson, M. N., Winter, C. C., \& Patterson, G. R. (2014). Coercive family process and early-onset conduct problems from age 2 to school entry. Development and Psychopathology, 26(4pt1), 917-932.

Stein, D. J., Phillips, K. A., Bolton, D., Fulford, K., Sadler, J. Z., \& Kendler, K. S. (2010). What is a mental/psychiatric disorder? From DSM-IV to DSM-V. Psychological Medicine, 40(11), 1759-1765.

Stier, M. (2013). Normative preconditions for the assessment of mental disorder. Frontiers in Psychology, 4, 611.

Szasz, T. S. (1960). The myth of mental illness. American Psychologist, 15(2), 113.

Szasz, T. S. (1963). Law, liberty, and psychiatry: An inquiry into the social uses of mental health practices: Syracuse University Press.

Szasz, T. S. (1974). The myth of mental illness: Foundations of a theory of personal conduct, Rev: Harper \& Row. 
Thompson, E. (2007). Mind in Life: Biology, Phenomenology, and the Sciences of Mind: Harvard University Press.

Thompson, E. (2011). Reply to commentaries. Journal of Consciousness Studies, 18(5-6), 176-223.

Thompson, E., \& Cosmelli, D. (2011). Brain in a vat or body in a world?: Brainbound versus enactive views of experience. Philosophical topics, 39(1), 163-180.

Thompson, E., \& Stapleton, M. (2009). Making sense of sense-making: Reflections on enactive and extended mind theories. Topoi, 28(1), 23-30.

Troisi, A., \& McGuire, M. T. (2002). Darwinian psychiatry and the concept of mental disorder. Neuroendocrinology Letters, 23(Suppl 4), 31-38.

Varela, F. J., Thompson, E., \& Rosch, E. (2017). The embodied mind: Cognitive science and human experience: MIT press.

Wakefield, J. C. (1992). The concept of mental disorder: On the boundary between biological facts and social values. American Psychologist, 47(3), 373.

Wakefield, J. C. (1997). Normal inability versus pathological disability: Why Ossorio's definition of mental disorder is not sufficient. Clinical Psychology: Science and Practice, 4(3), 249-258. 